ISSN 1112-9867

http://www.jfas.info

\title{
INTELLIGENCE QUOTIENT AND PERCEPTUAL ABILITY: AN INTER-RELATIONSHIP BASED ON BRAINWAVE POWER RATIO FEATURES
}

\author{
N. H. R. Azamin ${ }^{1}$, A. H. Jahidin ${ }^{2}$, M. S. A. Megat Ali ${ }^{1, *}$ and M. N. Taib ${ }^{1}$ \\ ${ }^{1}$ Faculty of Electrical Engineering, Universiti Teknologi MARA, Shah Alam, Selangor, \\ Malaysia \\ ${ }^{2}$ Centre for Foundation Studies in Science, University of Malaya, Kuala Lumpur, Malaysia
}

Published online: 10 November 2017

\begin{abstract}
Cognitive ability refers to the characteristic approach by the brain in processing information. These can be observed through various aspects of cognition such as intelligence and perceptual ability. Studies have shown that both mental constituents originate from the same neurological substrate in the prefrontal cortex. Hence, the paper discusses the relationship between intelligence and perceptual ability using electroencephalogram (EEG) features. The study is based on resting brainwave of fifty samples and focused on the left and right prefrontal cortex. The intelligence quotient (IQ) scores obtained are then used to establish the control groups. Subsequently, the pattern of alpha and theta power ratio for each IQ level is observed and successfully correlated with perceptual ability through the Neural Efficiency Hypothesis of intelligence.
\end{abstract}

Keywords: EEG; intelligence; IQ; perceptual ability; power ratio.

Author Correspondence, e-mail: megatsyahirul@salam.uitm.edu.my doi: http://dx.doi.org/10.4314/jfas.v9i6s.70 


\section{INTRODUCTION}

Cognitive ability, generally assessed in terms of intelligence is defined as the characteristic approach by the brain in processing information [1]. It is defined as the ability to comprehend complex ideas, efficiently adapt to the surroundings, involved in various forms of reasoning, solve problems and learn from experience [2]. Individual differences in intelligence are measured as intelligence quotient (IQ) [1]. It is the most robust observation in psychology and has the highest estimation of hereditability of human behavioral characteristics [3]. Among the various psychometric assessment schemes, Raven's Progressive Matrices (RPM) provides the optimum domain-independent measure of general intelligence [4]. Studies have also shown a correlation between intelligence and brain activity through the Neural Efficiency Hypothesis, which states that brighter individuals demonstrate efficient use of brain areas [1]. Meanwhile, perception is defined as the ability to interpret and process sensory information from the surroundings [5]. The ability is highly associated with attentional mechanism [6] which can be observed through the alpha band [7]. Hence, perceptual ability can be predicted by the strength of neuronal synchronization; a behavior that is commonly observed in the resting brain [8].

Evidences have strongly shown that cognitive ability is associated with attentional processes through the neural-transmission error and cerebral arousability theories. Studies have confirmed that subjects with higher IQ level demonstrate higher probability of error-less transmissions due to the well-functioning excitatory and inhibitory neurotransmitters. The low constant probability of transmission errors result in smaller deviation of rhythmic EEG [9] and are reflected in higher alpha power [10]. Concurrently, intelligent individuals exhibit low cerebral arousability due to inhibition of brainstem to external excitations. The low energy expenditure is further demonstrated by lower glucose metabolic rate in intelligent individuals [2]. Consequently, all these conform to the Neural Efficiency Hypothesis which states that the brain of intelligent individuals is less active than the less gifted ones [1].

The alpha suppression theory further elaborates the role of alpha waves in sustaining attention. Peak frequency of approximately $10 \mathrm{~Hz}$ is a setting state against which phasic changes occur. The baseline condition sets an active mechanism that leads to inhibition of task-irrelevant 
cortical activity and maintaining attention [11]. Therefore, higher alpha power not only reflects lower cortical noise but also increased perceptual limits [12]. These correlate to the ability of brilliant individuals that have shorter reaction time compared to the less intelligent ones [13]. However, synchronization of theta oscillations will lead to the de-synchronization of alpha waves [14]. Hence, the lower alpha power reflects higher noise level in the cerebrum which results in reduced perceptual limit and attentional state [15]. The behavior of both theta and alpha oscillations forms a reciprocal relationship, in which higher alpha power leads to lower theta power, and vice versa.

The executive functions of the brain; inclusive of cognitive abilities are performed by the prefrontal cortex. The left side is specialized for sequential and logic processes. Meanwhile, the right side of prefrontal cortex is more involved in emotion and social interaction capabilities [16]. It has also been established that both intelligence and attentional mechanism share a common neurological substrate in the prefrontal cortex. Hence, the paper elaborates the inter-relationship between IQ and perceptual ability based on brainwave patterns. The study will focus on power ratio features in alpha and theta bands.

\section{METHODOLOGY}

The methods comprise of data collection and EEG acquisition, signal pre-processing, feature extraction and evaluation of alpha and theta power ratio features for the high, medium and low IQ groups based on theories relating to perceptual ability. Fig. 1 shows the experimental framework that has been adopted in this study.

\subsection{Data Collection and EEG Acquisition}

50 university students from various fields have volunteered for data collection. The age range is between 18 to 40 years with mean age of 23.9 years and standard deviation of \pm 3.5 years. All subjects are right-handed, healthy and not under prescribed medications. Prior to data collection, the participants are briefed on the procedures and have completed the consent form. Subjects are required to seat in relaxed position with both eyes closed. Resting EEG is then acquired using g.MOBIlab ${ }^{+}$from the left (Fp1) and right prefrontal cortex (Fp2). The positioning of unipolar electrodes on the forehead conforms to the International 10-20 System 
for electrode placements. Each recording session lasts for approximately 3 minutes. The sampling rate of the device is $256 \mathrm{~Hz}$. Subsequently, the signal is analyzed offline in MATLAB [17, 23].

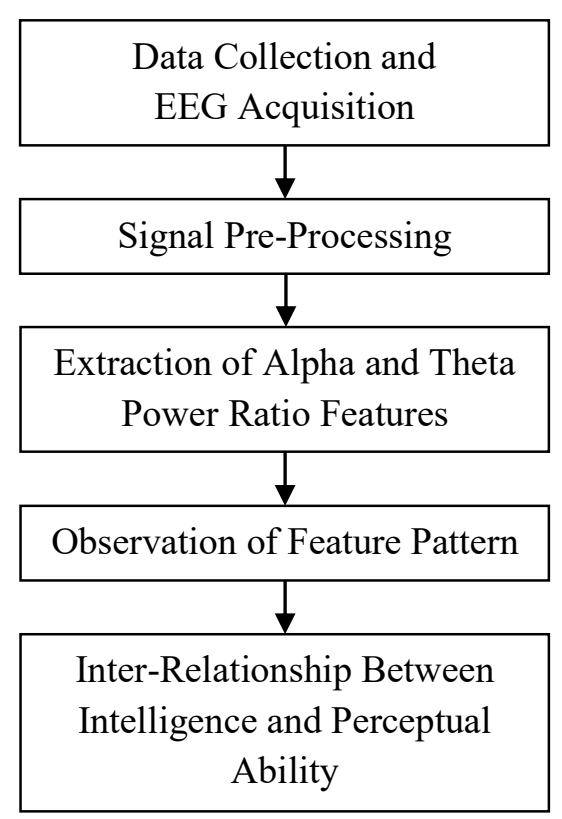

Fig.1. Framework of research methods

Subjects are then required to answer the online RPM. The IQ scores range from 0 to 150 . Subsequently, samples are grouped into high, medium and low IQ levels based on mean and standard deviation of the normally distributed scores. Table 1 summarizes the number of samples for each IQ group that were obtained from a related study. The obtained mean is 96.9 with standard deviation of 24.8 [17].

Table 1. IQ levels and number of samples

\begin{tabular}{cc}
\hline IQ Level & Number of Samples \\
\hline High & 6 \\
Medium & 39 \\
Low & 5 \\
\hline
\end{tabular}

\subsection{Signal Pre-Processing}

The raw EEG is initially filtered from direct current offset using band-pass equiripple filters. Signal epochs exceeding $\pm 100 \mu \mathrm{V}$ are considered as electrooculogram artefact , and thus are removed through automatic rejection method. The pre-processed signal is then limited to a 
standardized duration of 2 minutes 30 seconds. Subsequently, the artefact-free EEG is filtered into alpha and theta waves using equiripple band-pass filters. The filtered signals are then transformed into power spectral density (PSD) using Welch technique. Hamming window is implemented with 50\% overlapping epochs. Energy spectral density (ESD) for alpha and theta bands is obtained as the area under the respective PSD curve [18]. Power ratio method is then implemented to normalize the ESD between the related EEG [21] bands. The approach has proven effective in several applications involving characterization of IQ [17] and learning styles from brainwaves [19], as well as electrocardiogram in post-myocardial infarction conditions [20]. Alpha and theta power ratio is each expressed by Equation (1) and (2), where $\alpha$ is alpha ESD and $\theta$ is theta ESD.

$$
\begin{aligned}
& \text { Alpha Power Ratio }=\frac{\alpha}{\alpha+\theta} \\
& \text { Theta Power Ratio }=\frac{\theta}{\alpha+\theta}
\end{aligned}
$$

The features are segregated into the respective IQ groups and the pattern of median is analyzed using IBM SPSS Statistics.

\section{RESULTS AND DISCUSSION}

\subsection{Pattern of Alpha and Theta Power Ratio Features}

Fig. 2 and Fig. 3 each show the box plot for alpha power ratio from locations Fp1 and Fp2.

The pattern is similar for both sides of the prefrontal cortex.

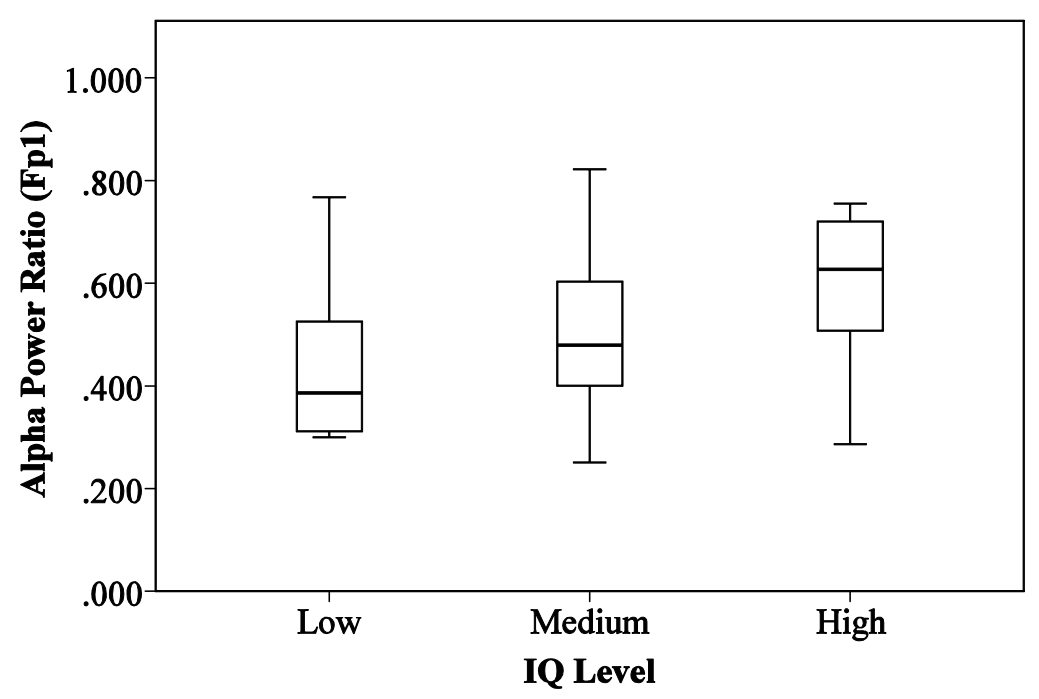

Fig.2. Box plot for alpha power ratio from the left prefrontal cortex 


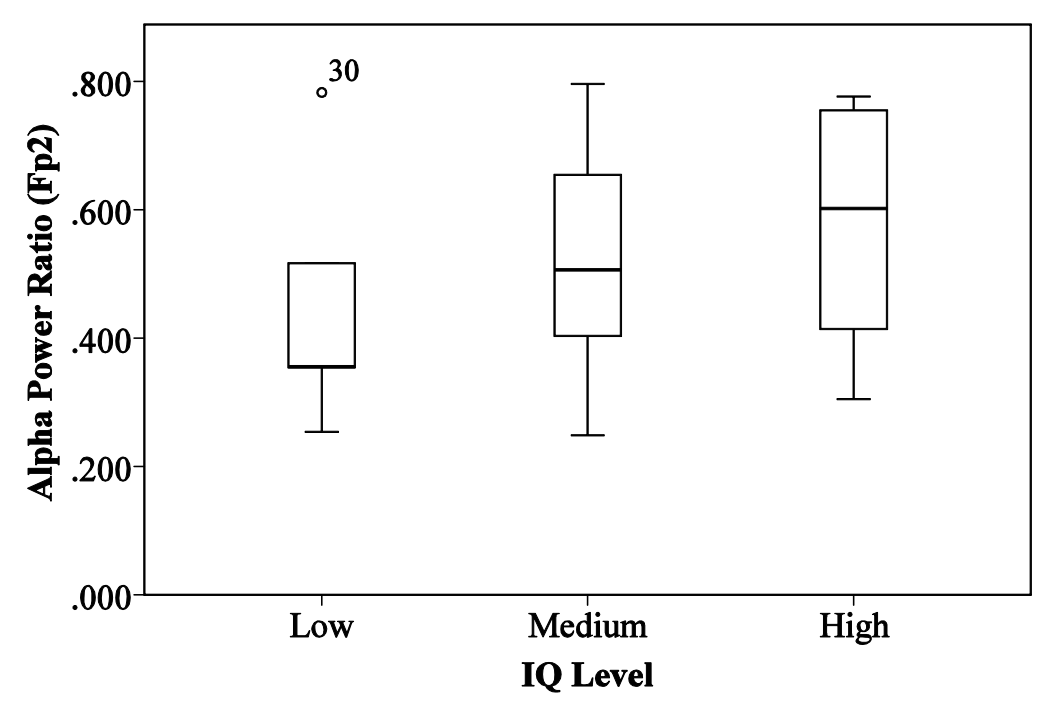

Fig.3. Box plot for alpha power ratio from the right prefrontal cortex

Meanwhile, one outlier has been observed for the low IQ group. The increasing median of alpha power ratio from low to high IQ group shows that the brain of gifted individuals is less active than the less intelligent ones. This conforms to the Neural Efficiency Hypothesis which states that brilliant individuals demonstrate efficient use of the cerebrum by disengaging it from irrelevant cortical activities.

Conversely, Fig. 4 and Fig. 5 each show the box plot for theta power ratio from left and right prefrontal cortex. Similar to the alpha power ratio feature; only one outlier has been identified from the low IQ group and the pattern is similar for both Fp1 and Fp2. The median of theta power ratio decreases with increasing IQ level. Hence, the results demonstrate an inversed pattern to that of alpha power ratio.

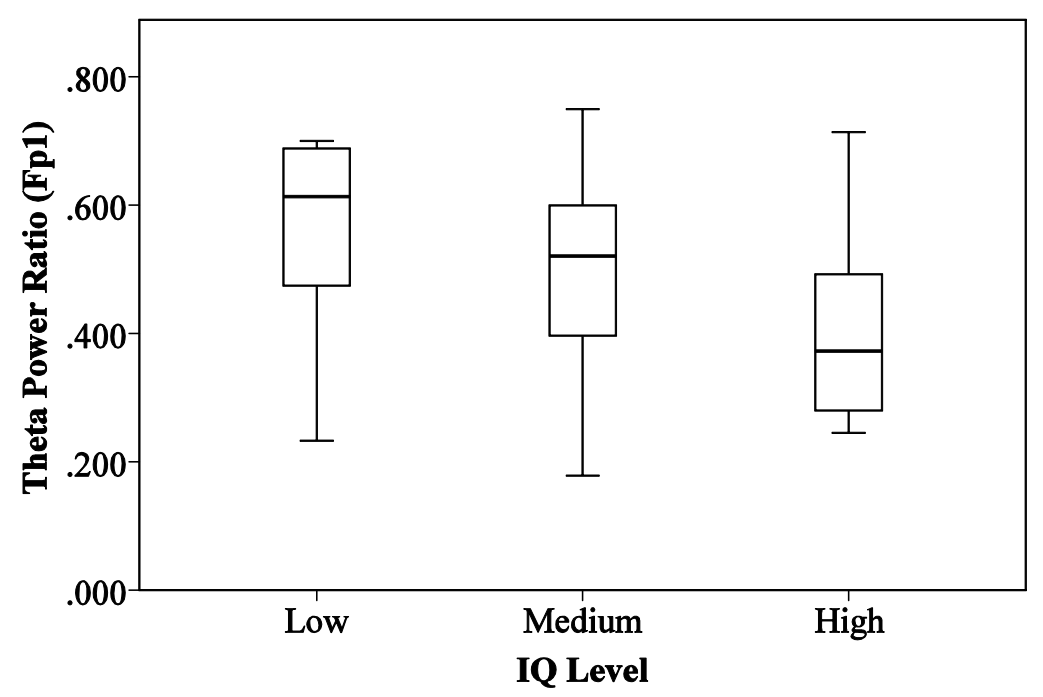

Fig.4. Box plot for theta power ratio from the left prefrontal cortex 


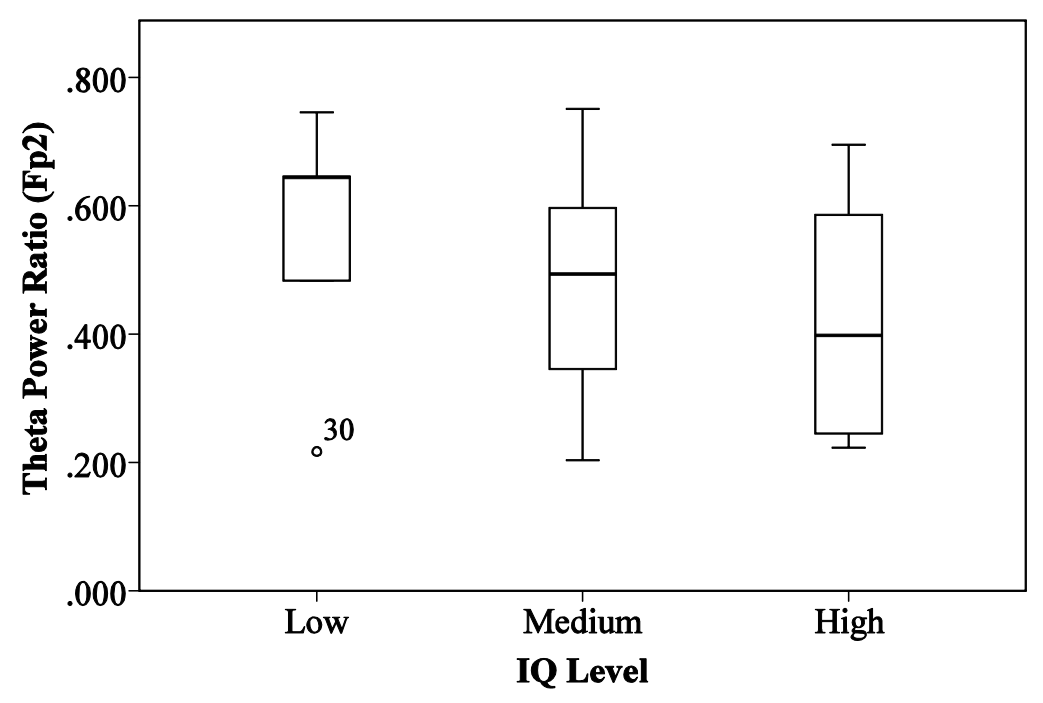

Fig.5. Box plot for theta power ratio from the right prefrontal cortex

The findings are considered valid as in tonic condition, the synchronization of alpha waves de-synchronizes theta oscillation, and vice versa. Hence, alpha power is inversely correlated with theta power.

\subsection{Relationship between IQ and Perceptual Ability based on Brainwave Pattern}

The pattern of alpha power ratio suggests that the high IQ group reveal smaller deviation of rhythmic EEG [22] due to the well-functioning excitatory and inhibitory neurotransmitters. Hence, intelligent individuals exhibit lower cerebral arousability due to the inhibition of brainstem from external excitations. The higher alpha power serves as an active mechanism that leads to inhibition of task-irrelevant cortical activity to sustain attention. Therefore, the high IQ group demonstrates higher level of perceptual ability, followed by medium and low IQ groups. Conversely, the higher theta power ratio shown by the low IQ group suggests higher cortical noise and reduced level of perceptual ability. These can also be correlated with past findings where brilliant individuals demonstrate shorter reaction time compared to the less intelligent ones.

\section{CONCLUSION}

The study has successfully shown that IQ and perceptual ability can be related via brainwave pattern. Investigations through alpha and theta power ratio have provided conclusive evidence in which IQ and perceptual ability is positively correlated. While preliminary findings shows conformity with the Neural Efficiency Hypothesis of intelligence, further investigation 
provides validation that intelligent individuals exhibit lower cortical noise and the synchronized alpha oscillation inhibits task-irrelevant cortical activities which leads to sustained attention and consequently, higher level of perceptual ability.

\section{ACKNOWLEDGEMENTS}

The work is supported by the Ministry of Education, Malaysia (600-RMI/FRGS 5/3 (63/2015)) and Universiti Teknologi MARA (600-IRMI/MyRA 5/3/LESTARI (015/2017)).

\section{REFERENCES}

[1] Neubauer A C, Fink A. Intelligence and neural efficiency. Neuroscience and Biobehavioral Reviews, 2009, 33(7):1004-1023

[2] Haier R J, Siegel B, Tang C, Abel L, Buchsbaum M S. Intelligence and changes in regional cerebral glucose metabolic rate following learning. Intelligence, 1992, 16(3):415-426 [3] Lutzenberger W, Birbaumer N, Flor H, Rockstroh B, Elbert T. Dimensional analysis of the human EEG and intelligence. Neuroscience Letters, 1992, 143(1):10-14

[4] Gray J R, Thompson P M. Neurobiology of intelligence: Science and ethics. Nature Reviews Neuroscience, 2004, 5(6):471-482

[5] Zhang L F. Field-dependence/independence: Cognitive style or perceptual ability? Validating against thinking styles and academic achievement. Personality and Individual Differences, 2004, 37(6):1295-131

[6] Robertson L, Lamb M, Knight R. Effects of lesions of temporal-parietal junction on perceptual and attentional processing in humans. Journal of Neuroscience, 1988, $8(10): 3757-3769$

[7] Klimesch W. Alpha-band oscillations, attention, and controlled access to stored information. Trends in Cognitive Sciences, 2012, 16(12):606-617

[8] Womelsdorf T, Fries P. The role of neuronal synchronization in selective attention. Current Opinion in Neurobiology, 2007, 17(2):154-160

[9] Fidelman U. Neural transmission-errors, cerebral arousability and hemisphericity: Some relations with intelligence and personality. Kybernetes, 1999, 28(6/7): 695-725 
[10] Jahidin A H, Taib M N, Md Tahir N, Megat Ali M S A, Lias S, Fuad N, Omar W R W. Brainwave sub-band power ratio characteristics in intelligence assessment. In IEEE Control and System Graduate Research Colloquium, 2012, pp. 318-321

[11] Dockree P M, Kelly S P, Foxe J J, Reilly R B, Robertson I H. Optimal sustained attention is linked to the spectral content of background EEG activity: Greater ongoing tonic alpha ( $10 \mathrm{~Hz}$ ) power supports successful phasic goal activation. European Journal of Neuroscience, 2007, 25(3):900-907

[12] Foxe J J, Snyder A C. The role of alpha-band brain oscillations as a sensory suppression mechanism during selective attention. Frontiers in Psychology, 2011, 2:1-13

[13] Jensen A R. Why is reaction time correlated with psychometric g? Current Directions in Psychological Science, 1993, 2(2):53-56

[14] Klimesch W. EEG alpha and theta oscillations reflect cognitive and memory performance: A review and analysis. Brain Research Reviews, 1999, 29(2-3):169-195

[15] Strijkstra A M, Beersma D G, Drayer B, Halbesma N, Daan S. Subjective sleepiness correlates negatively with global alpha $(8-12 \mathrm{~Hz})$ and positively with central frontal theta (4-8 $\mathrm{Hz}$ ) frequencies in the human resting awake electroencephalogram. Neuroscience Letters, 2003, 340(1):17-20

[16] van der Knaap L J, van der Ham I J M. How does the corpus callosum mediate interhemispheric transfer? A review. Behavioural Brain Research, 2011, 223(1):211-221

[17] Jahidin A H, Megat Ali M S A, Taib M N, Md Tahir N, Yassin I M, Lias S. Classification of intelligence quotient via brainwave sub-band power ratio features and artificial neural network. Computer Methods and Programs in Biomedicine, 2014, 114(1):50-59

[18] Megat Ali M S A, Taib M N, Md Tahir N, Jahidin A H, Yassin I M. EEG sub-band spectral centroid frequencies extraction based on Hamming and equiripple filters: A comparative study. In IEEE 10th International Colloquium on Signal Processing and its Applications, 2014, pp. 199-203

[19] Megat Ali M S A, Jahidin A H, Taib M N, Md Tahir N. EEG sub-band spectral centroid frequency and amplitude ratio features: A comparative study in learning style classification. Jurnal Teknologi, 2016, 78(2):15-23 
[20] Megat Ali M S A, Jahidin A H, Yassin I M, Pasya I, Khalid M F A, Awang Z. Characterization of post-MI electrocardiogram using power ratio features and k-nearest neighbor classifier. Journal of Fundamental and Applied Sciences, 2017, 9(4S):937-951

[21] Zaman F H, Shukur N A, Hamzah N, Zaini N M, Rizman Z I. Performance of modified power spectral density features in EEG signal classification. Journal of Fundamental and Applied Sciences, 2017, 9(3S):830-843

[22] Ismail W W, Hanif M, Mohamed S B, Hamzah N, Rizman Z I. Human emotion detection via brain waves study by using electroencephalogram (EEG). International Journal on Advanced Science, Engineering and Information Technology, 2016, 6(6):1005-1011

[23] Nawi B, Sulaini B, Mohd Z A, Shamsul A Z, Zairi I R. PID voltage control for DC motor using MATLAB Simulink and Arduino microcontroller. Journal of Applied Environmental and Biological Sciences, 2015, 5(9):166-173

\section{How to cite this article:}

Azamin N H R, Jahidin A H, Megat Ali M S A and Taib M N. Intelligence and perceptual ability: An inter-relationship based on brainwave power ratio features. J. Fundam. Appl. Sci., 2017, 9(6S), 944-953. 